\title{
Non-ICT Students' Familiarity with Basic Internet Services and Tools at the Faculty of Philosophy at the University in Novi Sad
}

The aim of this study was to collect and analyse data about the prior knowledge that freshmen at the Faculty of Philosophy at the University of Novi Sad, Serbia, have about basic concepts in computer and information literacy. The results can be used for a better adaptation of teaching to take into account the knowledge that students have accumulated during their primary and secondary education. The study relied on an anonymous testing of respondents' knowledge. The questions encompassed two base ECDL modules: the essentials of computer and Internet use. The test was completed by more than 500 students from 13 departments at the Faculty of Philosophy, one group from the Faculty of Management and one group from the Faculty of Technical Sciences. This paper analyses the students' answers to the questions regarding their knowledge of Internet services and tools. The results reveal significant deficiencies in their knowledge in this area.

Keywords: computer and information literacy, digital literacy, students, Internet

\section{Author Information}

Zoltán Gellér, Faculty of Philosophy, University of Novi Sad, Novi Sad, Serbia https://orcid.org/0000-0003-1853-4740

Tanja Jevremov, Faculty of Philosophy, University of Novi Sad, Novi Sad, Serbia https://orcid.org/0000-0003-3780-3424

Karlo Bala, Faculty of Philosophy, University of Novi Sad, Novi Sad, Serbia https://orcid.org/0000-0002-9267-236X

Dubravka Valić Nedeljković, University of Montenegro, Podgorica, Montenegro https://orcid.org/0000-0002-9729-9752

Mirjana Ivanović, Faculty of Sciences, University of Novi Sad, Novi Sad, Serbia https://orcid.org/0000-0003-1946-0384

\section{How to cite this article:}

Gellér, Zoltán, Tanja Jevremov, Karlo Bala, Dubravka Valić Nedeljković,

Mirjana Ivanović. "Non-ICT Students' Familiarity with Basic Internet Services and Tools at the Faculty of Philosophy at the University in Novi Sad".

Információs Társadalom XXI, no. 4 (2021): 38-66.

https://dx.doi.org/10.22503/inftars.XXI.2021.4.3

All materials

published in this journal are licenced

as CC-by-nc-nd 4.0 


\section{Introduction}

The teaching of informatics in elementary schools in Serbia was introduced only in the first decade of the third millennium as an elective subject with 36 lessons per year (1 lesson a week). The subject Informatics and Computing is studied from the fifth grade and envisages some acquaintance with basic concepts of operating systems, text processing and multimedia (The Official Gazette of the Republic of Serbia - Education Gazette no. 6/2007). In secondary schools, within the compulsory subject Computing and Informatics, these areas are further expanded and complemented with new elements, such as presentations and spreadsheets. However, this subject is not studied to the same extent in all secondary schools: while it is studied for four years in grammar schools, in vocational schools, it is often taught only in the first year (The Official Gazette of the Republic of Serbia - Education Gazette nos 6/2012, 5/2011, 4/2002, 8/1995).

Despite the fact that computer and information literacy (CIL) is one of the essential elements of functional literacy, without which survival in the modern world would become increasingly complicated, up until the end of 2017, there had been a continuous public discussion in Serbia about whether this subject should be considered compulsory or elective. It was considered that "digital natives" (Prensky 2001a) and (Presnky 2001b) do not need the subject since they have grown up in the digital environment, which means they would have been automatically acquiring this knowledge since early childhood.

Therefore, it was considered that digital natives by default would have a good grasp of basic computer literacy, even without receiving formal education in this field, but this is not quite true in practice. It has been noticed that, upon arrival at faculty, many students do not have sufficient pre-knowledge. In other words, they come with different levels of knowledge depending on their prior success in school, their preferences, and the competence of the teachers in the schools they had attended before entering the faculty.

According to the data at our disposal and to the best of our knowledge, there has been no prior research on the computer and information literacy of freshmen at the level of higher education in Serbia. At the same time, the needs for such data are indisputable, primarily because the data can be used to help make new curricula adequate to the prior knowledge, needs, and predicted outcomes of the students, as well as to help them acquire the new skills they need upon completion of related courses at the university.

This study, which was conducted by our team at the University of Novi Sad, Serbia (the Faculty of Philosophy in Novi Sad and with control groups from the Faculty of Technical Sciences and Faculty of Management), provides important information and instruction related to freshmen's levels of knowledge of computer and information literacy. This literacy is an important tool for mastering all other educational content during their studies and also plays a significant role in their digital day-to-day life, including in the context of their daily schedule/engagements. CIL along with digital literacy form the basis for introducing innovation in the learning process. This is precisely what numerous studies in the field of pedagogy and teaching methodology insist on. 
Khairnar states that "Teaching with technology engages students with different kinds of stimuli involved in activity based learning. Technology makes material more interesting. It makes students and teachers more media literate” (Khairnar $2015,869)$. The author argues that smart gadgets are especially important in the new approach to teaching. However, this is not possible without a solid basis of knowledge and skills in CIL and digital literacy.

Molnár and her team of assistants at the University of Szeged studied "students' attitudes and skills in solving problems using multimedia technologies and measured how students can integrate multimedia tools in the educational process and in the curriculum" (Molnár 2008, 44). The author concluded that students were typically ready for new experiences, skills, and knowledge if offered the opportunity.

In 2000, a team from Ohio University tested freshmen to determine what their real computer and information competence was. The study found that while the students themselves thought they were digitally literate, the results proved the contrary: "Only $9 \%$ of the Ohio State freshmen taking a three-part proficiency test achieved a passing score of $70 \%$ on the entire test. More of these students (30\%) passed the first part on use of Internet tools; their performance was poorest (16\%) on the second part, searching skills" (O'Hanlon 2002, 55).

Based on the results of researching the information and computer literacy of undergraduate and graduate LIS students, Jokić et al. concluded that "contrary to popular belief, there is no relationship between information and computer literacy, in other words, the students who possess information literacy do not necessarily possess computer literacy and vice versa" (Jokić et al. 2016, 89).

Heerwegh, De Wit and Verhoeven found that "the impact of ICT classes in secondary school is not apparent in the ICT skills of the students" (Heerwegh, De Wit and Verhoeven 2016, 36) and that the type of studies is an important variable, too: "The self-perceived proficiency in ICT skills is higher for science students who see computers as useful and necessary for scientific research" (Heerwegh, De Wit and Verhoeven 2016, 38).

Similar research was conducted in the Netherlands, but with three age groups of high school students (11-13,14-15, and 16-17). The authors concluded that, even in countries that are significantly better developed, "the level of information and strategic Internet skills among Dutch secondary students have much room for improvement” (van Deursen and van Diepen 2013, 223). The authors also concluded that, "Unfortunately, Internet skills in general and the acquisition of information and strategic skills in particular play a minor role in Dutch classes" (van Deursen and van Diepen 2013, 224). That finding is completely in accordance with the results of the long-standing research project: "How children realise their communicative needs", involving 10 to 14-year olds, that students and teachers from the Department of Media Studies at the Faculty of Philosophy at the University of Novi Sad conducted in the territory of Vojvodina (Serbia). The results indicated that children and teenagers, despite being able to use the computer, do not use the Internet as an aid in doing homework, nor for communication with their teachers about lessons and learning, but only for entertainment (Valić Nedeljković 2011; Valić Nedeljković 2012; Valić Nedeljković, Bala and Geler 2013). They lack basic information and com- 
puter knowledge and skills, which would otherwise help them understand that the Internet can be used to gain new knowledge. Also, their teachers were not prone to introducing digital innovations in their teaching.

Van Deursen and van Diepen concluded that "New educational materials should be developed that are designed for Internet use and implemented in existing courses of the school curriculum. When the development of information and strategic skills is implemented in existing courses, such as language, history, biology and geography, it is likely that they will improve" (van Deursen and van Diepen 2013, 224), since this is more appropriate to the specific traits of millennials, who represent a generation of digital natives. A new requirement for these new educational materials is high CIL and digital competency among students and teachers. In order to achieve this, it is important to establish the real level of students' knowledge beforehand, because CIL and digital literacy are what equips them to perform the tasks and activities imposed by the technical and technological progress of the third millennium they are growing up in.

In their study of the general Internet usage patterns of undergraduate students, Deniz and Geyik also argue that finding information online is useful for students, and they concluded that "spending long hours during online might be helpful for [the] young to make some contributions to their knowledge about information technologies and unlike some people's prejudices, surfing [the] Internet is not totally [a] waste of time for students but more or less a productive facility” (Deniz and Geyik 2015, 895).

Slechtova states that “students' willingness to use ICT for studying and attitudes to e-learning are not homogenous and show [a] certain relation to their field of study and computer skills. The characteristics of digital natives do not apply to everyone, which should be reflected in courses using ICT in higher education institutions" (Slechtova 2015, 1128).

In addition, according to Duţă and Martínez-Rivera, "students recognize the need and importance of ICT as a tool for collaborative learning and as we see there are great similarities between them about training methods, their application is mainly aimed at seminars on the use of multimedia equipment teaching-learning-assessment by some teachers and students, [it is] thought-provoking that there is an area that teachers are not fluent enough in Romania and therefore, the request is in line with this. It is essential to consider these views to develop proposals for improvement in the near future.” (Duţă and Martínez-Rivera 2015, 1472).

Lekka and Pange state that teachers' expectations are greater than the students' knowledge: "many students are not able to keep up to date. On the other hand, teachers expect their students to have excellent technology capabilities and to use ICT tools in their everyday assignments. This expectation may turn into reality if and only if, teachers use ICT tools well-known to students. This way they will offer numerous opportunities to students for collaboration, inventiveness, and active learning” (Lekka and Pange 2015, 388).

At the end of this brief review of the available literature related to the research focus of this paper, it should be noted that, as Serdyukov says, "In education, efficiency of learning is determined mainly by the invested time and cost. Learning 
is more efficient if we achieve the same results in less time and with less expense. Productivity is determined by estimating the outcomes obtained vs the invested effort in order to achieve the result. Thus, if we can achieve more with less effort, productivity increases. Hence, innovations in education should increase both [the] productivity of learning and [the] learning efficiency" (Serdyukov 2017, 8).

Today, in the third millennium, there is no efficient learning without the use of digital technologies in the educational process at all levels, especially without solid computer and information literacy as the basis for that learning. This basis requires the curricula to be designed in a modern way, which can only be created on the basis of the availability of a clear picture of the level of students' computer and information literacy in a given time and space. The research and results presented in this paper provide an answer to that requirement.

The rest of the paper is organised as follows. The second section presents the corpus and the methodology used. A detailed overview of the students' answers to the test questions and statistical analysis of the collected data are given in the third section. The last section is devoted to the discussion of the results.

\section{Study Corpus and Methodology}

The focus of the research of this paper is first-year students' basic computer literacy, related to their knowledge of basic Internet concepts, regarding students at the Faculty of Philosophy at the University of Novi Sad, Serbia. The testing included more than 500 first-year students from 13 out of 17 study programmes (only the programmes with a small number of students were not covered). In addition, testing was also performed with a group of students from the Faculty of Management and a group of students from the Faculty of Technical Sciences, also at the University of Novi Sad, as control groups and with the aim to gain better insights into the nature of the collected results. The numbers of freshmen from each study programme who took the test are shown in Table $1^{1}$. The total number of freshmen who participated in the research was 568, of whom, 522 were from the Faculty of Philosophy and 46 were students from the control groups.

The test used for knowledge evaluation comprised 22 closed-ended questions with 4 options per question. Of the 22 questions, 14 refer to the most basic computer literacy concepts and 8 to basic Internet terms. The questions were extracted from the set of questions used for compiling the final tests for the Computer Literacy subject at the Faculty of Philosophy. This paper provides an overview and analysis of the answers to the eight questions about the basic Internet terms. The students were tested at the beginning of lessons in agreement with the subject teachers, whose cooperation enabled the testing to proceed. The tests were distributed in print and the participants answered the questions anonymously. The students had no access to computers during the testing and were given 15-20 minutes to complete the test.

\footnotetext{
${ }^{1}$ For the sake of a clearer presentation of the results, the short labels given in the first column of the table will be used further in this paper.
} 


\begin{tabular}{c|c|c|c}
\hline Label & Study programme & $\begin{array}{c}\text { Number of } \\
\text { respondents (N) }\end{array}$ & $\begin{array}{c}\text { Total number } \\
\text { of freshmen }\end{array}$ \\
\hline JRN & Journalism & 52 & 70 \\
HIS & History & 57 & 69 \\
PSY & Psychology & 61 & 80 \\
HUN & Hungarian Language and Literature & 12 & 16 \\
PHI & Philosophy & 38 & 60 \\
SOC & Sociology & 40 & 55 \\
CL & Comparative Literature & 14 & 20 \\
SER & Serbian Language and Literature & 31 & 50 \\
GER & German Language and Literature & 49 & 60 \\
ENG & English Language and Literature & 40 & 70 \\
FRE & French Language and Literature & 20 & 47 \\
PED & Pedagogy & 57 & 75 \\
SL & Serbian Literature & 51 & 71 \\
FTN & Faculty of Technical Sciences & 29 & \\
FAM & Faculty of Management & 17 & \\
\hline FF & Faculty of Philosophy & 522 & \\
TOTAL & & 568 & \\
\hline
\end{tabular}

Table 1. Study programmes that participated in the testing and the numbers of respondents

\subsection{Data analysis}

The scores of the students from the Faculty of Philosophy (FF) were evaluated and compared with the scores of the students in the control groups from the Faculty of Technical Sciences (FTN) and Faculty of Management (FAM). The students' answers were analysed both with regard to the total score on the test and for the individual questions. The overall scores of the students from the FF for all the study programmes and for each study programme individually were also analysed.

The answers given by the students from the FF and from the control groups from the FTN and FAM to each question are graphically presented below. The statistical significance for the correlation between different faculties, individual study programmes within the FF, and the number of correct answers was tested by Pearson's $\chi 2$ test. The level of association is expressed by Cramer's V coefficient. Distributions of correct answers for the FTN, FAM, and the individual study programmes in the FF were also analysed. Measures of central tendency and variability were calculated. In order to gain insights into the students' success, their pass rates on the European Computer Driving Licence (ECDL) test and in faculty exams were also analysed. 
Differences in the scores of the students from the FF in relation to the control groups were tested by using the Kruskal-Wallis test and z-tests for contrasting the differences between the average ranges with a Bonferroni adjustment. These nonparametric tests based on the sums of ranges were chosen due to the observed nonhomogeneous distribution of the results of the tested groups.

The last step of the analysis was the calculation of the phi coefficients of correlation between the questions in order to test the relation between the test questions with regard to the number of correct answers.

\section{Results}

As a first level presentation of the test results, an overview and graphical representation of the students' answers to the individual test questions are given in the form of charts, as shown below. In these charts, the percentage of students who did not answer the given question is marked by an X. The complete test can be found in Appendix A. The short labels, which are later used in the tables to mark the questions, can be found in brackets after the question numbers. A short explanation of the used terms and offered answers is provided before each question. For better orientation, the correct answers in the list of possible answers are marked in bold. Based on the statistical analysis of the first-year students' answers, the second level describes the general picture of their familiarity with the covered basic Internet concepts.

\subsection{Overview of the students' answers}

Question 1 (INET). The goal of the first question was to test whether students know what the Internet represents, i.e. whether and to what degree they identify it with its best-known, most-visible, and most-frequently used service - the system of mutually connected hypertextual documents known by the abbreviation the "web". The following options were possible for the answer to the question: The Internet is ... (the correct answer is 2. global computer network):

1. World Wide Web

2. global computer network

3. social network

4. type of server

As can be seen in Chart B in Figure 1, while the majority of students (55.6\%) gave the correct answer, a significant percentage (44.4\%) of the first-year students at the FF gave wrong answers; for instance, 39.1\% (204 out of the 522 respondents) identified the Internet with the web (Chart A). Looking at Chart D, it is clear that the FF students' answers were similar to those of the students from the FAM, while the results for the group of students from the FTN were significantly better, whereby almost $80 \%$ of the students answered the question correctly (Chart C). The results also indicated that the students could differentiate between the concepts of social networks and the Internet. 


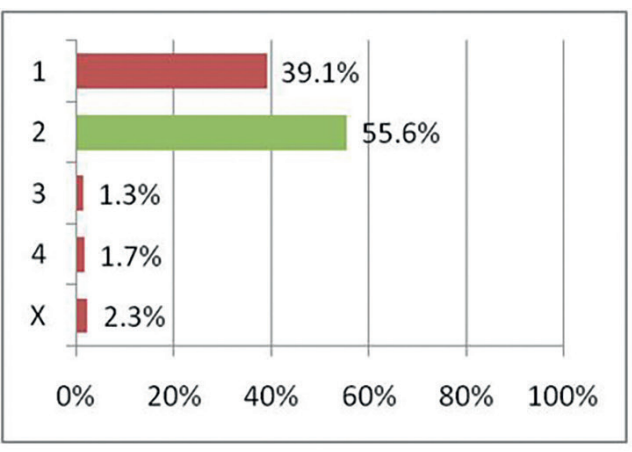

A

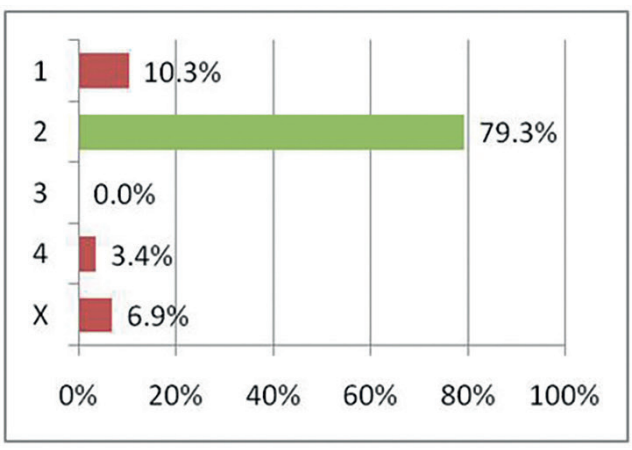

C

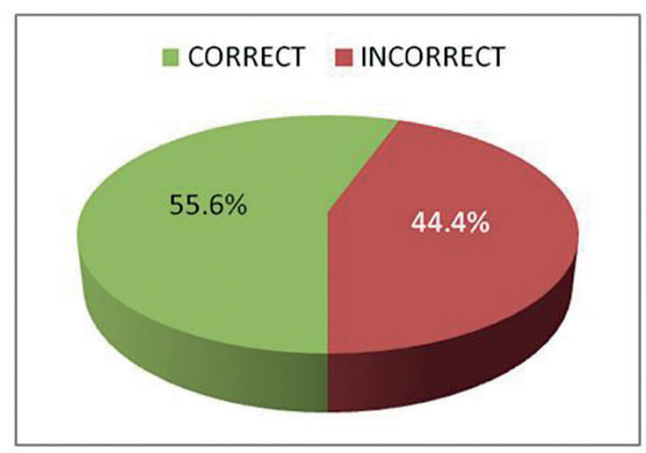

B

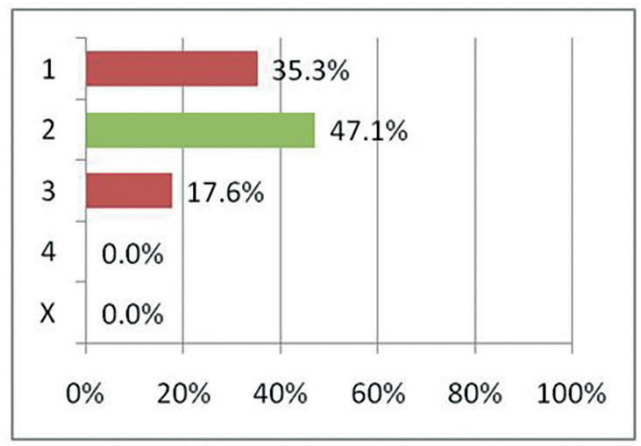

D

Figure 1. Percentage representation of answers to the first question by the students from the FF (A), a group of students from the FTN (C), and a group of students from the FAM (D); ratio of incorrect to correct answers by the students from the FF (B).

Question 2 (IP). Data transfer on the Internet relies on a set of protocols known as TCP/IP. TCP (Transmission Control Protocol) ensures reliable data exchange between computers in the form of small data packages called datagrams. The role of the IP (Internet Protocol) is to address the networked devices and to direct data. The second test question checked whether students could recognise the abbreviation IP (correct answer, 3. Internet Protocol) among the following alternatives:

1. Internet Store (Ser. Internet Prodavnica)

2. Internet Provider

3. Internet Protocol

4. Internet Presentation

Chart B in Figure 2 clearly indicates that almost $2 / 3$ (64.9\%, or 339 of 522) of the tested students from the FF wrongly identified the acronym IP. Almost $60 \%$ of respondents thought that the abbreviation IP refers to the provider of Internet services, i.e. Internet Provider - answer 2 (see Chart A). As was the case in the first question, the situation with the group of students from the FAM was again similar (Chart D). The percentage of correct answers in the FTN group was again close 
to $80 \%$, amounting to $76 \%$ (Chart C). Interestingly, very few students in any of the groups chose the other two answers (Internet Store and Internet Presentation).

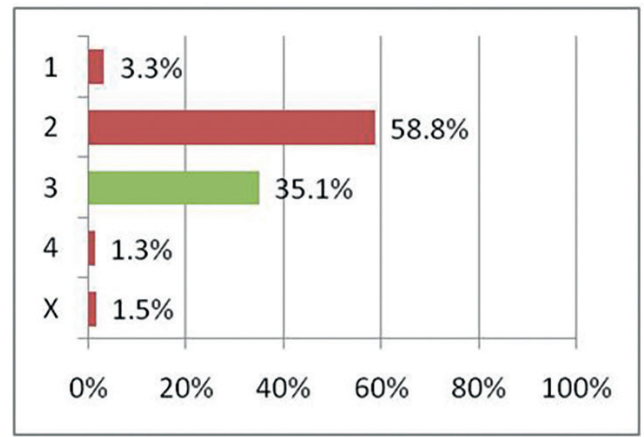

A

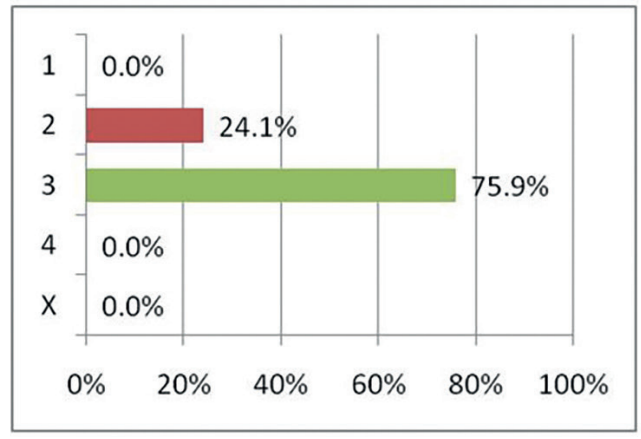

C

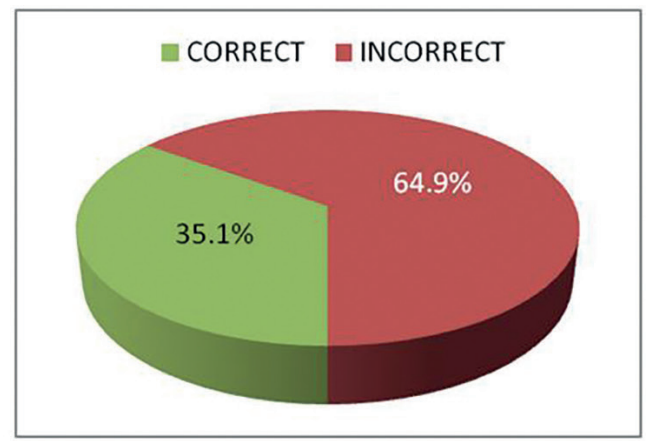

B

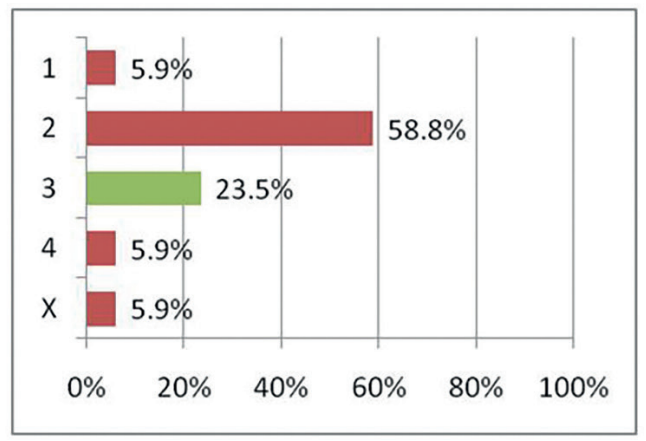

D

Figure 2. Percentage representation of answers to the second question by the students from the FF (A), a group of students from the FTN (C), and a group of students from the FAM (D); ratio of incorrect to correct answers by the students from the FF (B).

Question 3 (LAN). Regarding their size (covered area), computer networks can be divided into two main categories: a local network (LAN - Local Area Network), which covers a relatively small area, such as a classroom, office, or building; or a wide regional network (WAN - Wide Area Network), which covers a wider area, such as a town, region, or country. The third question on the test aimed to check students' familiarity with these terms and abbreviations, which are integral parts of a globally connected world. Their task was to identify what type of network a LAN is among the following categories (the correct answer is 2 . covers a relatively small area (e.g. office, classroom, building)):

1. covers a wider area (e.g. town, region)

2. covers a relatively small area (e.g. office, classroom, building)

3. connects distant servers

4. is a global computer network

Comparing Figures 3 and 1, it can be noticed that the results for the first and third questions were quite similar. In both cases, almost half the students from the FF 
chose a wrong answer (see Chart B). The most frequent wrong answer was 1 . covers a wider area (e.g. town, region) (18.6\%), closely followed by the third answer, 3. connects distant servers (15.5\%), which actually represents a non-existent, imaginary category of computer networks. A number of respondents (8.8\%) chose the fourth answer, 4. is a global computer network, which is actually the definition of the Internet. While the percentages of wrong answers from the FAM group (Chart D) did not significantly differ from these results, impressively, nearly $90 \%$ of the students from the FTN circled the correct answer (see Chart C).

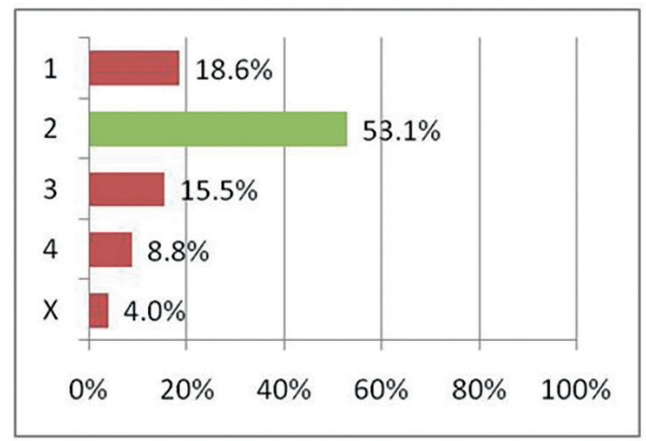

A

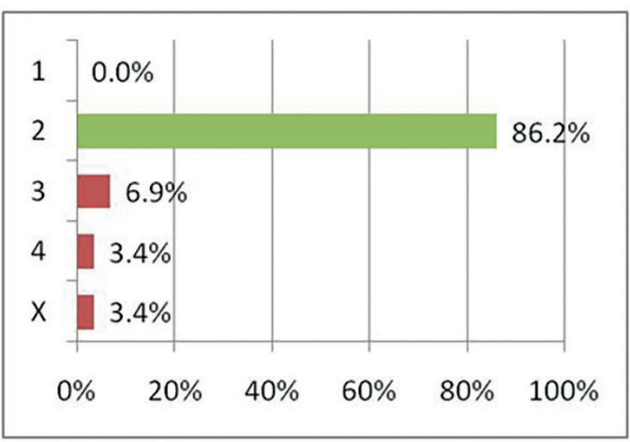

C

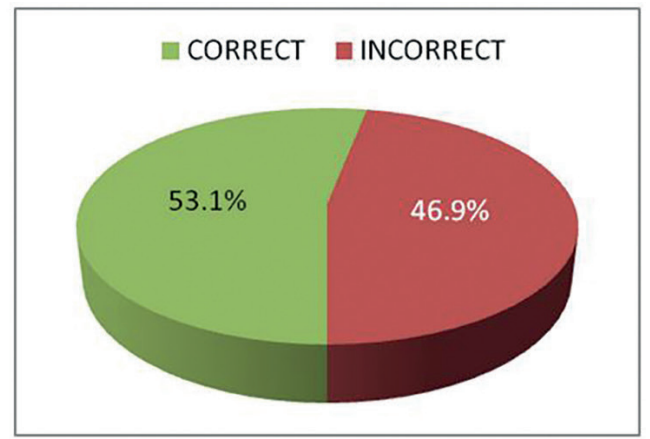

B

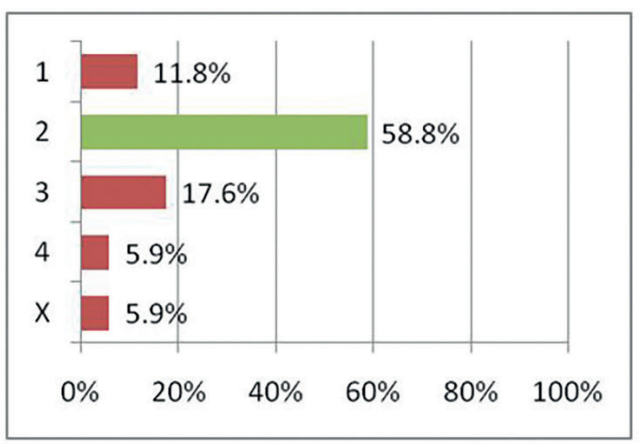

D

Figure 3. Percentage representation of answers to the third question by the students from the FF (A), a group of students from the FTN (C), and a group of students from the FAM (D); ratio of incorrect to correct answers by the students from the FF (B).

Question 4 (URL). The URL (Uniform Resource Locator) address is one of the key Internet concepts and represents a unique address for resources, such as web pages, documents, programs, pictures, videos, and other data forms. For example, the web page containing the exam schedules at the FF can be accessed through the URL address: http://www.ff.uns.ac.rs/sr-lat/studenti/rasporedi/raspored-ispita/osnovne-studije. In the fourth question, the students were asked what URL represents and were offered the following answers (the correct answer is 4. unique address of Internet resources):

1. transfer protocol for web pages

2. numerical label that uniquely identifies every computer in a network 


\section{3. domain name}

4. unique address of Internet resources

Here, the first three answers can be integral parts of a URL address. Looking at the provided example of a URL address, the protocol for transferring web pages is http (HyperText Transfer Protocol). The domain name is www.ff.uns.ac.rs and it represents an understandable letter substitute for a numerical label that uniquely identifies the computer on which the exam schedules are located. This numerical label is called the IP address and is used in internal communication in a computer network.

Based on Chart B in Figure 4, it can be seen that most (60\%) of the tested students from the FF were familiar with the concept of a URL address. Though charts A, C, and $\mathrm{D}$ indicate that some respondents confused this term with the protocol for transferring web pages, as well as less often with the domain name. The category of IP address was the rarest choice on the test, which probably indicated that the students do not deal with IP addresses directly. Interestingly, the percentage of correct answers in the FTN group was not significantly higher than the results for the students from the FF.

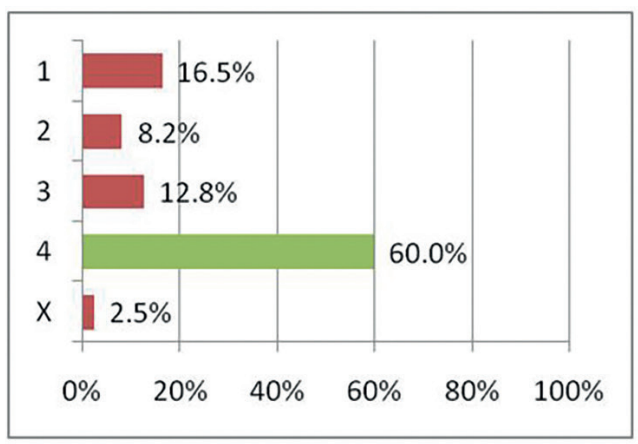

A

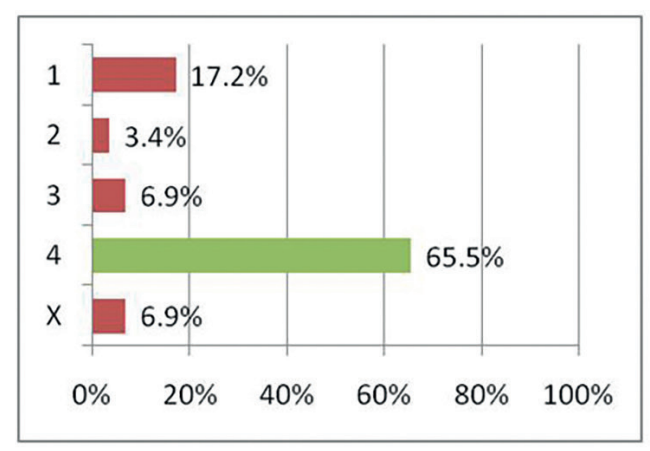

C

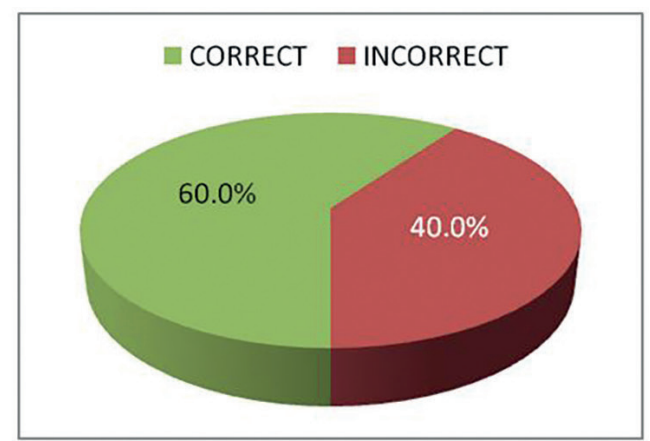

B

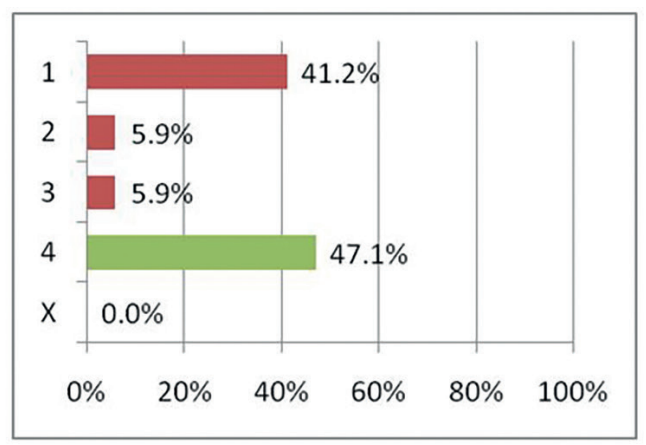

D

Figure 4. Percentage representation of answers to the fourth question by the students from the FF (A), a group of students from the FTN (C), and a group of students from the FAM (D); ratio of incorrect to correct answers by the students from the FF (B). 
Question 5 (ORG). A domain name is the part of a URL address that represents the name of a website that somebody wants to visit. For example, in the case of the URL address http://www.ff.uns.ac.rs/sr-lat/studenti/rasporedi/raspored-ispita/osnovne-studije, which contains the exams schedules at the FF, the domain name is www.ff.uns.ac.rs. The part of a domain name after the last dot is called the top-level domain (in this case .rs) and indicates the kind of domain and type of website. Apart from top-level national domains such as .rs, which also indicate countries, there are several international domains that are important to know for getting by in the Internet world. The fifth test question checked whether students could recognise these international domains. From the following options, the respondents were asked to choose the top-level domain that indicates the websites of non-profit organisations (the correct answer is 3. ORG):
1. $\mathrm{COM}$
2. NET
3. ORG
4. XLSX

The percentage of wrong answers by the students from the FF was nearly $60 \%$ (Chart B in Figure 5), which shows that most of them were not familiar with the meanings of these labels.

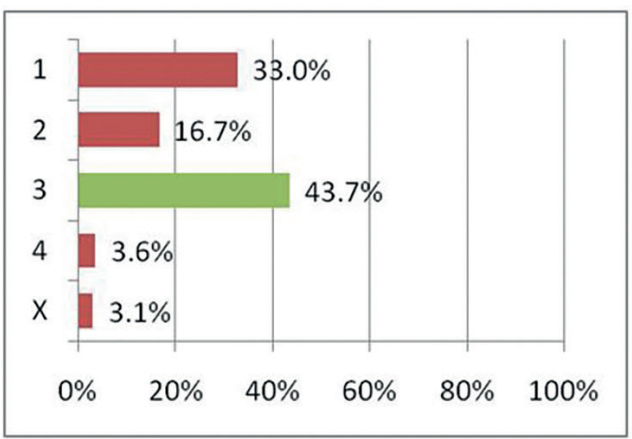

A

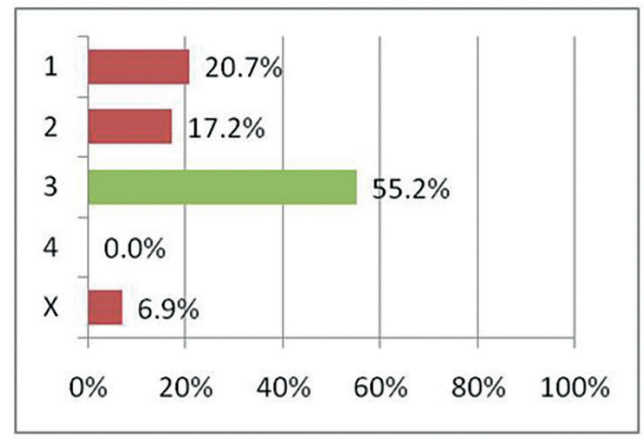

C

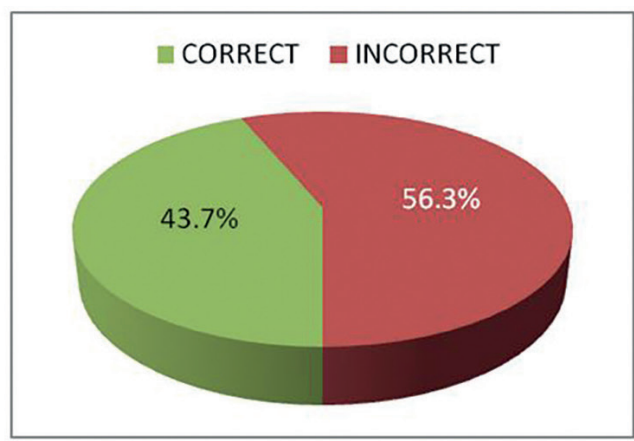

B

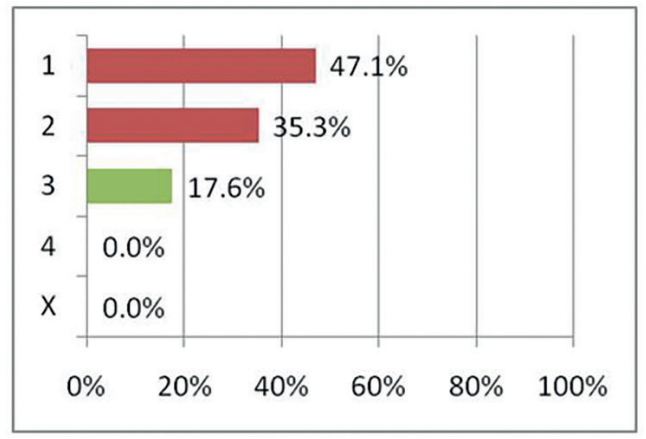

D

Figure 5. Percentage representation of answers to the fifth question by the students from the FF (A), a group of students from the FTN (C), and a group of students from the

FAM (D); ratio of incorrect to correct answers by the students from the FF (B). 
Special attention should be paid to the fact that the most common among the wrong answers was the first option, 1. COM, i.e. the domain used by commercial organisations (see Charts A, C, and D in Figure 5). The question arises, to what extent is this the consequence of the fact that popular and seemingly free Internet services, such as the search engine Google or the social network Facebook, which have grown into unavoidable components of everyday Internet experience, can be found with addresses in the COM domain?

The domain NET, which primarily labels the locations of commercial organisations that deal with computer networking (i.e. Internet service providers), was also noticeably present among the wrong answers: $17 \%$ of students linked this domain with non-profit organisations.

While Chart D in Figure 5 indicates that the domain ORG was largely an unknown category among the students in the FAM group, Chart $\mathrm{C}$ shows that the situation with the respondents in the FTN group was better, but even in that group almost half the students (44.8\%) gave a wrong answer.

Also, the results clearly show that the students could correctly recognise that the fourth category (4. XLSX) did not belong to the Internet world, which was correct as XLSX does not represent a domain, but rather is a file extension of Excel workbooks.

Question 6 (HTML). HTML (HyperText Markup Language) is the language for the markup of hypertext (web pages) and a basic tool for creating web content. HTML is used for the markup of different parts of web pages, such as paragraphs, titles, pictures, and links. Web pages are kept on computers in files with the extension (type) .html. The sixth question on the test tested the students' knowledge of what the name of the language used for creating web pages is and the following options were offered (the correct answer is 2. HyperText Markup Language):

1. World Wide Web

2. HyperText Markup Language

3. HTTP

4. Hypertext

The first answer, 1 . World Wide Web, is the full name of the Internet's best-known service, i.e. the Web. The third answer, 3. HTTP, is the acronym of the protocol for transferring hypertext (web pages) through the Internet (HyperText Transfer Proto$\mathrm{col}$ ) and the fourth, 4. Hypertext, is hypertext in English. In a general sense, hypertext means a document that contains connections (links) to parts of the document itself and/or to other documents - in the Internet world, these are web pages themselves.

The sixth question was the most technologically oriented part of the test, which is reflected in the results shown in Figure 6. The percentage of wrong answers among the students from FF was the highest in the case of this question, reaching $70 \%$ (Chart B). Nearly half the respondents (44.6\%) marked HTTP (protocol for transferring web pages) as the language for making web pages. This response predominated in the FAM group as well (Chart D) and it was markedly present in the FTN group, although most students in that group (slightly over 60\%) answered the question correctly. The similarity between the acronyms HTML and HTTP probably contributed to this result. 


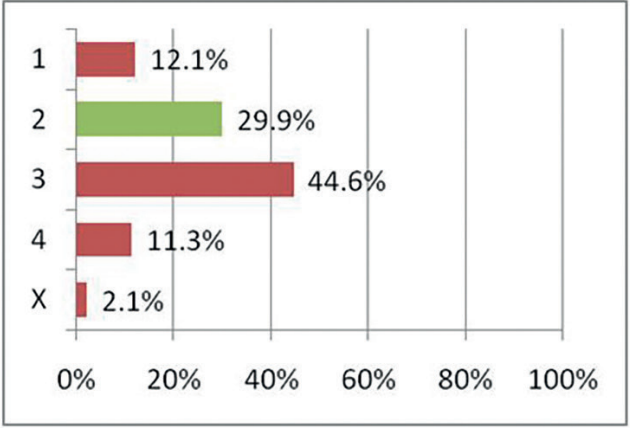

A

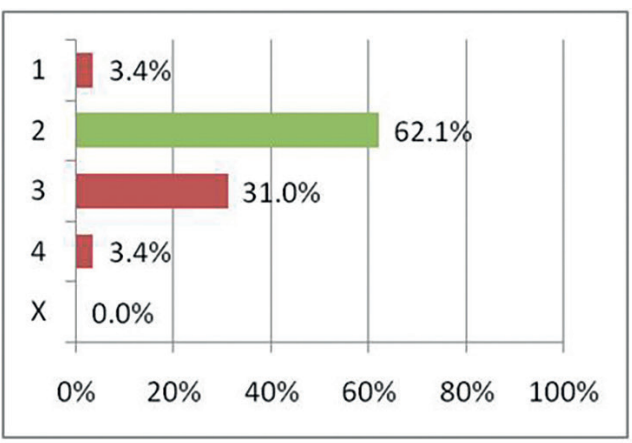

C

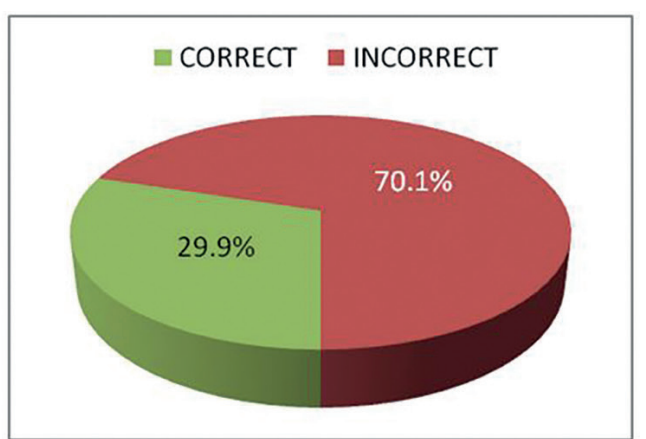

B

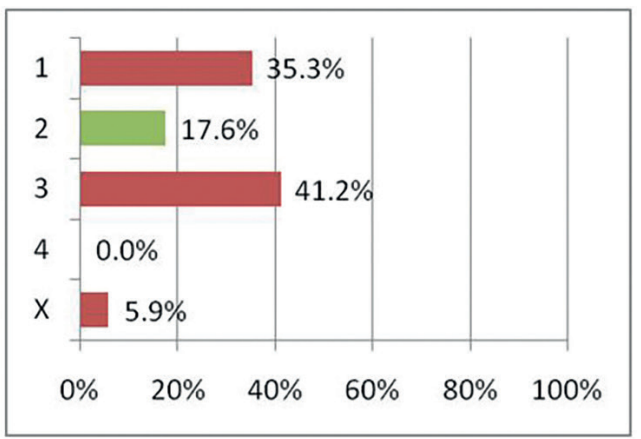

$\mathrm{D}$

Figure 6. Percentage representation of answers to the sixth question by the students from the FF (A), a group of students from the FTN (C), and a group of students from the FAM (D); ratio of incorrect to correct answers by the students from the FF (B).

Question 7 (IE). Web pages created with HTML language are opened by entering their URL addresses in programs called web browsers, which are created for that specific purpose. There are many different web browsers available, the most popular being Google Chrome, Safari, Firefox, Opera, and Internet Explorer, with the latter being part of the Windows operating system. The options offered in the penultimate question included three programs and one web service (Google Mail), the task being to choose which is a web browser (the correct answer is 3 . Internet Explorer):

\section{Google Mail \\ 2. Windows Explorer \\ 3. Internet Explorer \\ 4. Microsoft FrontPage}

Based on the results presented in Figure 7, it is evident that this task did not cause any difficulties for most the participants. Around $85 \%$ of students from the FF correctly identified Internet Explorer as the program for visiting websites, and the other answers were only marginally present (Chart A). Similar results were noticed in the FAM and FTN groups (Charts D and C, respectively). 


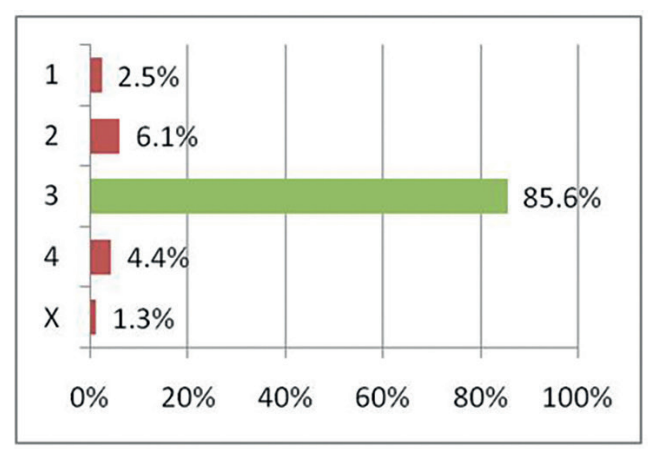

A

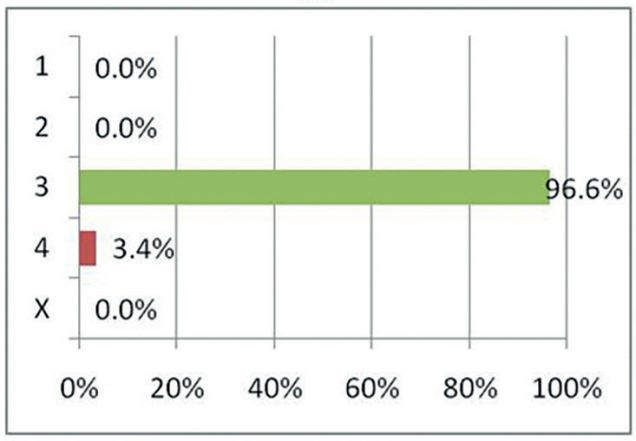

C

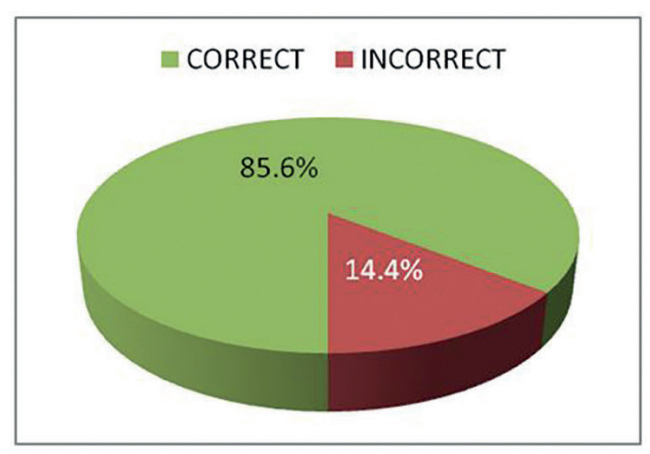

B

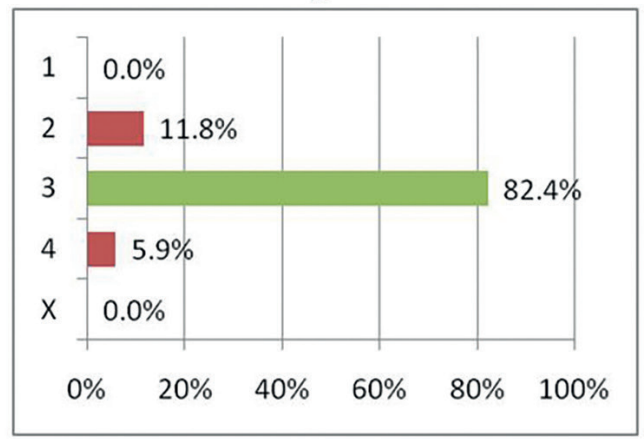

D

Figure 7. Percentage representation of answers to the seventh question by the students from the FF (A), a group of students from the FTN (C), and a group of students from the

FAM (D); ratio of incorrect to correct answers by the students from the FF (B).

Question 8 (MAIL). Since electronic mail is an unavoidable part of formal Internet communication, the last question was posed with the aim of discovering whether the students were familiar with popular email clients, in this case specifically Thunderbird. Apart from the Thunderbird program, possible answers included the web browser Chrome, the previously popular instant messaging client Google Talk (support for which had already ended at the time of the research), and a social network, i.e. the microblogging service Twitter (the correct answer is 1. Thunderbird):
1. Thunderbird
2. Chrome
3. Google Talk
4. Twitter

This structure of possible answers was determined with the awareness that the respondents all belong to a generation that is predominantly oriented towards the use of online services, social networking sites, and instant messages.

Noticeably, there was a high percentage $(65.7 \%)$ of wrong answers (Chart B in Figure 8), which indicated that the first-year students in the FF most probably rely on online services, such as Google Mail, for communication by electronic mail and that they are not familiar with specialised programs. Interestingly, a large num- 
ber (almost 42\%) chose Google Talk, a specialised program for instant messaging (Chart A) - maybe while thinking of Google Mail? The online-orientation of respondents may also account for the fact that a noticeable percentage (nearly 17\%) chose the Chrome web browser (which can be used for accessing online email services). Similar results were noticed in the groups of students from the FTN (Chart C) and FAM (Chart D).

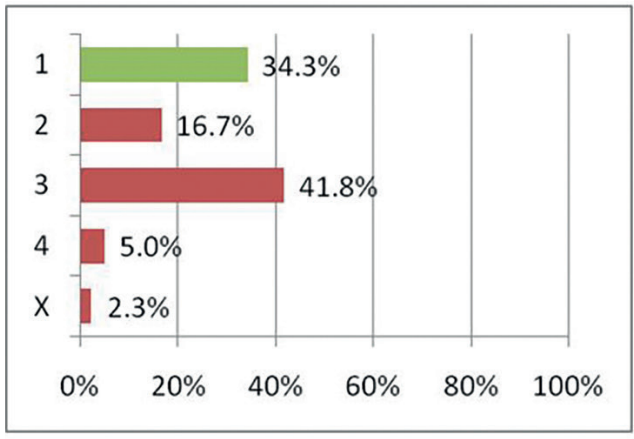

A

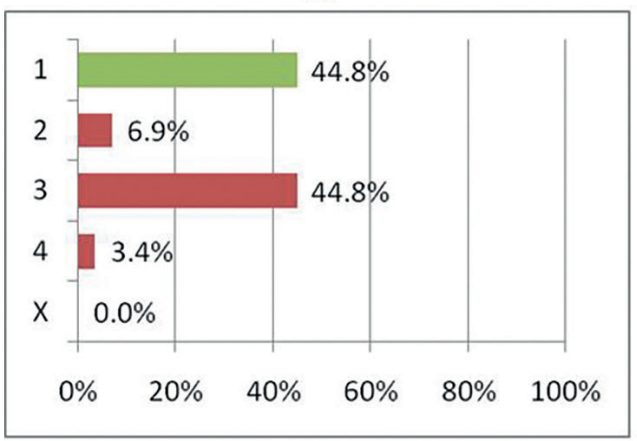

C

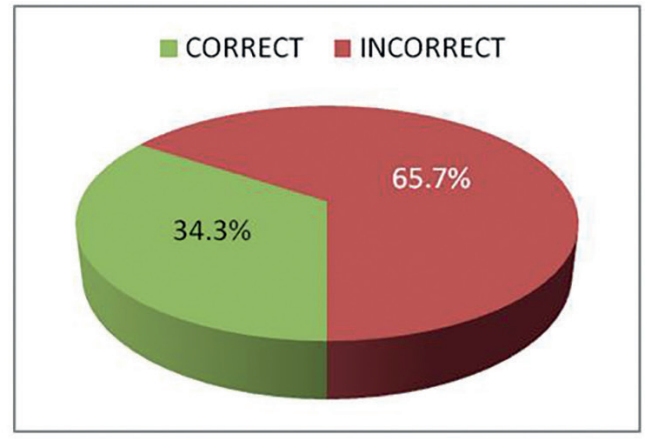

B

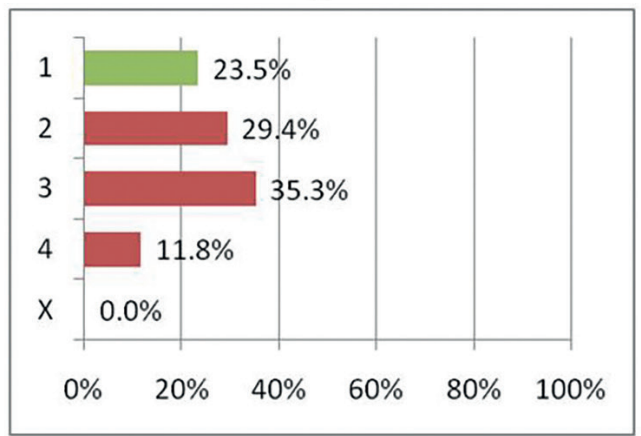

D

Figure 8. Percentage representation of answers to the eighth question by the students from the FF (A), a group of students from the FTN (C), and a group of students from the

FAM (D); ratio of incorrect to correct answers by the students from the FF (B).

\subsection{Percentages of correct answers}

The percentages of correct answers per question (columns) and study programmes are shown in Table 2 . The best results for each question are marked by the symbol $\bullet$ and the worst with the symbol $\circ$. For example, for the first question (INET), the best result was achieved by the FTN group (79.3\%) and the worst by the FRE group (40\%). The column TEST shows the aggregate results for all the test questions. The best results per study programme are marked in bold and the worst are underlined. For example, the students in Journalism (JRN) achieved the best result in the question IE (94.2\%) and the worst in the question MAIL (23.1\%). Row FF shows the aggregate 
results for the students from the Faculty of Philosophy, and the row ALL shows the aggregate results of all the students who participated in the testing.

As for the students from the Faculty of Philosophy, based on row FF in Table 2, it is evident that among all the basic Internet concepts, the one they were most familiar with was the concept of the web browser (85.6\% of correct answers) and their least familiar was the concept of the HTML language for the creation of web pages (29.9\% of correct answers). Looking at the set of all the answers given for the eight test questions by the students from the FF, the ratio of correct to incorrect answers was approximately $50 \%$.

\begin{tabular}{|c|c|c|c|c|c|c|c|c|c|}
\hline & INET & IP & LAN & URL & ORG & HTML & IE & MAIL & TEST \\
\hline JRN & 69.2 & 34.6 & 50.0 & 61.5 & 48.1 & 30.8 & 94.2 & $\underline{23.1}$ & 51.4 \\
\hline HIS & 52.6 & 38.6 & 56.1 & 35.1 & 35.1 & 40.4 & 82.5 & $\underline{17.5} \circ$ & 44.7 \\
\hline PSY & 60.7 & 44.3 & 65.6 & 78.7 & 52.5 & $\underline{31.1}$ & 91.8 & 45.9 & 58.8 \\
\hline HUN & 58.3 & 33.3 & 41.7 & $\underline{8.3} \circ$ & $16.7 \circ$ & $\underline{8.3} \circ$ & 100.0 & 25.0 & $36.5 \circ$ \\
\hline PHI & 55.3 & $\underline{42.1}$ & 47.4 & 50.0 & 47.4 & $\underline{42.1}$ & 86.8 & $\underline{42.1}$ & 51.6 \\
\hline SOC & 50.0 & $\underline{17.5} \circ$ & 47.5 & 80.0 & 45.0 & 30.0 & 82.5 & 32.5 & 48.1 \\
\hline CL & 64.3 & 57.1 & 78.6 & 50.0 & 64.3 & $\underline{21.4}$ & 85.7 & $\underline{21.4}$ & 55.4 \\
\hline SER & 77.4 & $\underline{19.4}$ & 45.2 & 58.1 & 41.9 & 35.5 & 87.1 & 32.3 & 49.6 \\
\hline GER & 44.9 & 30.6 & 51.0 & 55.1 & 36.7 & 24.5 & 89.8 & 38.8 & 46.4 \\
\hline ENG & 47.5 & 67.5 & 57.5 & 67.5 & $70.0 \bullet$ & $\underline{37.5}$ & 95.0 & $72.5 \bullet$ & 64.4 \\
\hline FRE & $40.0 \circ$ & $\underline{20.0}$ & 60.0 & $100.0 \bullet$ & 55.0 & 40.0 & 90.0 & 35.0 & 55.0 \\
\hline PED & 57.9 & 26.3 & $33.3 \circ$ & 49.1 & $\underline{17.5}$ & 22.8 & 73.7 & 22.8 & 37.9 \\
\hline SL & 47.1 & 27.5 & 64.7 & 66.7 & 47.1 & $\underline{13.7}$ & $70.6 \circ$ & 31.4 & 46.1 \\
\hline FTN & $79.3 \bullet$ & $75.9 \bullet$ & $86.2 \bullet$ & 65.5 & 55.2 & $62.1 \bullet$ & 96.6 & $\underline{44.8}$ & $70.7 \bullet$ \\
\hline FAM & 47.1 & 23.5 & 58.8 & 47.1 & $\underline{17.6}$ & $\underline{17.6}$ & 82.4 & 23.5 & 39.7 \\
\hline FF & 55.6 & 35.1 & 53.1 & 60.0 & 43.7 & $\underline{29.9}$ & 85.6 & 34.3 & 49.6 \\
\hline ALL & 56.5 & 36.8 & 54.9 & 59.9 & 43.5 & $\underline{31.2}$ & 86.1 & 34.5 & 50.4 \\
\hline MIN & 40.0 & 17.5 & 33.3 & 8.3 & 16.7 & 8.3 & 70.6 & 17.5 & 36.5 \\
\hline MAX & 79.3 & 75.9 & 86.2 & 100.0 & 70.0 & 62.1 & 100.0 & 72.5 & 70.7 \\
\hline
\end{tabular}

Note: $\bullet$ denotes the best and $\circ$ the worst result for each question (column). The best result of each study group (row) is marekd in bold and the worst one is underlined.

Table 2. Percentage of correct answers for each group 
In order to observe the answering trend more easily, this data is visually presented using charts in Figure 9. As can be seen in Chart B in this figure, the average success rate of students from the FF was between that of the FTN and FAM students. Chart A in Figure 9 and the numerical results in Table 2 show differences in the variability of the results for the various study groups with regard to the questions. For some questions, the study programmes have quite homogenous results; e.g. a high percentage of students in all the programmes answered correctly the question about web browsers (IE), while all scored below average $(<50 \%)$ for the question about HTML, except for the FTN group, and all scored below average $(<50 \%)$ for the question about electronic mail (MAIL), except for the English Language and Literature (ENG) group. The highest variability between groups was regarding the percentage of correct answers to the question about URL, which was answered correctly by all the students in FRE, but less than $10 \%$ of students in HUN.

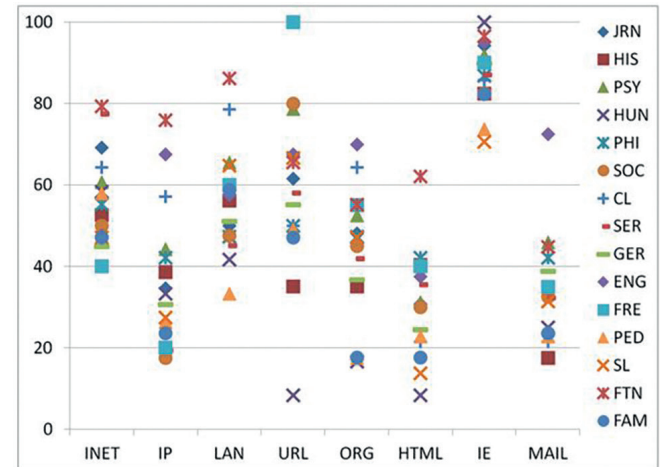

A

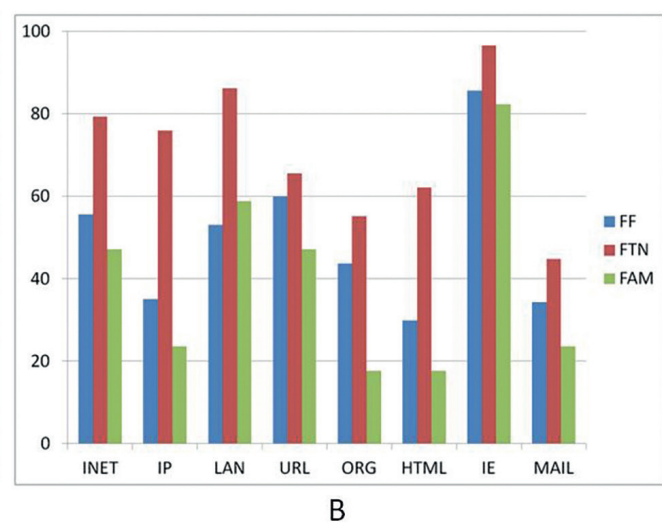

B

Figure 9. Graphical representation of the percentage of correct answers by students from the study programmes of the FF (A) and from different faculties (B)

\subsection{Statistical significance of the differences in the answers among the groups}

Table 3 shows the results of the $\chi 2$ testing which was used to test differences in the correctness of the answers among the study programmes at the Faculty of Philosophy (A) and the differences in the correctness of the answers among the faculties (B). Column V contains the obtained Cramer's V coefficients. At the significance level of $\alpha=0.05$, this analysis indicated that the relation between the number of correct answers and study programmes in the FF was not statistically significant, only in the cases of the INET and HTML questions. Looking at the relation between the number of correct answers and the faculties, it can be concluded that there was no statistically significant difference in the cases of the URL, IE, and MAIL questions, while a mild statistically significant difference was detected in the case of the ORG question. 


\begin{tabular}{|c|c|c|c|c|c|c|}
\hline & \multicolumn{3}{|c|}{$\begin{array}{c}\text { A } \\
\mathrm{df}=12, \mathrm{~N}=522\end{array}$} & \multicolumn{3}{|c|}{$\begin{array}{c}\text { B } \\
\mathrm{df}=2, \mathrm{~N}=568\end{array}$} \\
\hline & $\chi^{2}$ & $\mathbf{p}$ & $\mathbf{V}$ & $\chi^{2}$ & $\mathbf{p}$ & $\mathbf{V}$ \\
\hline INET & 18.634 & 0.098 & 0.189 & 6.946 & 0.031 & 0.111 \\
\hline IP & 39.324 & 0.000 & 0.274 & 20.996 & 0.000 & 0.192 \\
\hline LAN & 22.767 & 0.030 & 0.209 & 12.296 & 0.002 & 0.147 \\
\hline URL & 64.391 & 0.000 & 0.351 & 1.548 & 0.461 & 0.052 \\
\hline ORG & 39.614 & 0.000 & 0.275 & 6.238 & 0.044 & 0.105 \\
\hline HTML & 19.838 & 0.070 & 0.195 & 14.758 & 0.001 & 0.161 \\
\hline IE & 27.761 & 0.006 & 0.231 & 2.940 & 0.230 & 0.072 \\
\hline MAIL & 46.166 & 0.000 & 0.297 & 2.284 & 0.319 & 0.063 \\
\hline
\end{tabular}

Table 3. Results of the $\chi 2$ tests and Cramer's $V$ coefficients of the relationships between answer correctness and the study programmes at the FF (A) and among the faculties (B)

\subsection{Distributions of answers}

\begin{tabular}{ccccccccc}
\hline & N & Mean & SD & Median & Q25 & Q75 & Min & Max \\
\hline JRN & 52 & 4.12 & 1.69 & 4 & 3 & 5 & 0 & 8 \\
HIS & 57 & 3.58 & 1.81 & 3 & 2 & 5 & 1 & 8 \\
PSY & 61 & 4.70 & 1.43 & 5 & 4 & 6 & 2 & 8 \\
HUN & 12 & 2.92 & 1.16 & 3 & 2.25 & 3.75 & 1 & 5 \\
PHI & 38 & 4.13 & 1.68 & 4 & 3 & 5 & 0 & 8 \\
SOC & 40 & 3.85 & 1.56 & 4 & 3 & 5 & 0 & 7 \\
CL & 14 & 4.43 & 1.16 & 4.5 & 3 & 5.25 & 3 & 6 \\
SER & 31 & 3.97 & 1.45 & 4 & 3 & 5 & 2 & 7 \\
GER & 49 & 3.71 & 1.55 & 4 & 2.5 & 4 & 1 & 7 \\
ENG & 40 & 5.15 & 1.93 & 5 & 4 & 7 & 2 & 8 \\
FRE & 20 & 4.40 & 1.43 & 4 & 4 & 4 & 2 & 8 \\
PED & 57 & 3.04 & 1.66 & 3 & 2 & 4 & 0 & 7 \\
SL & 51 & 3.69 & 1.49 & 4 & 3 & 5 & 0 & 6 \\
FTN & 29 & 5.66 & 1.74 & 6 & 5 & 7 & 2 & 8 \\
FAM & 17 & 3.18 & 0.95 & 3 & 2 & 4 & 2 & 5 \\
\hline
\end{tabular}

Table 4. Measures of the central tendency and variability of the correct answers of the study programmes 
Table 4 shows the values obtained for the indicators of the central tendency and variability of the total number of correct answers for the students in the FTN and FAM groups and in the individual study programmes at the FF.

The results show that the scores of all the analysed groups of students from the FF were worse than the FTN students, to a lesser or greater extent. The only groups whose average score was somewhat lower than the FAM students' scores were the students in HUN and PED. Apart from this, the results showed relatively great differences between the study programmes at the FF in relation to the average achievement. Of the eight possible answers, which is the number of questions in the test, the average number of correct answers varied between 2.92 (for the groups HUN and PED) and 5.15 for the group ENG. Relatively great individual differences between students' answers were also evident; whereby, while some of them answered all the questions correctly, some did not give any correct answers to any question. Furthermore, there were differences in the variability of the achieved results between the analysed groups. It could be noticed that the results for the students from certain programmes with a small number of respondents were the most similar. Those are the groups in which most students did quite poorly (HUN and FAM) or had average scores (CL and FRE). The groups ENG and HIS, which were characterised by a greater variability of results in comparison to the other groups, had larger numbers of tested students and somewhat asymmetric distributions, which indicated that the variability was increased partly due to the smaller number of high results. This asymmetry of certain distributions can be seen in Figure 10, where the boxplots (box and whisker plots) show the medians, interquartile ranges, and lowest and highest observations.

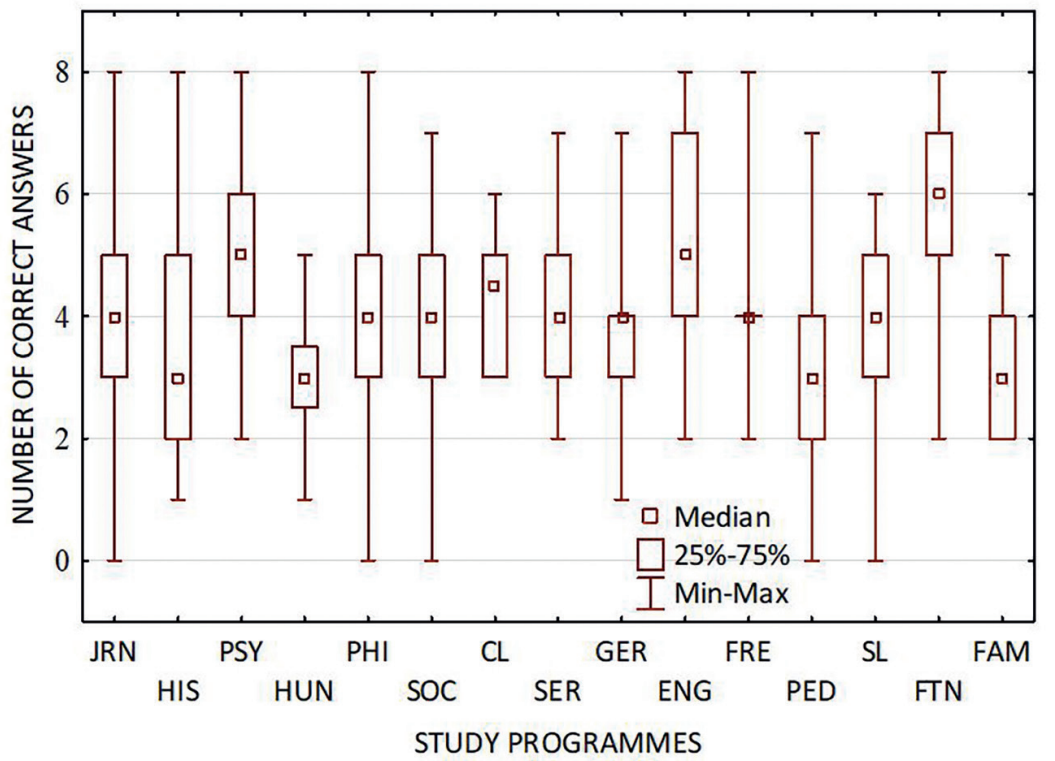

Figure 10. Boxplots (box \& whisker plots) of the central values and variability in the scores of the student groups considering the total number of correct answers 
The described differences in the number of correct answers between students in the different study programmes (FTN, FAM, and individual groups in the FF) were corroborated by the Kruskal-Wallis test, whose value was H(14, N = 568) $=84.728, \mathrm{p}<$ 0.001 . The z-test showed that these differences were a consequence of the better results achieved by the FTN students in comparison to a large number of the other analysed groups (SOC, GER, SL, HIS, FAM, PED, HUN); better results achieved by the ENG and PSY students in comparison to the HIS, FAM and PED students; and better results achieved by the students of ENG compared to the HUN students (Table 7 in Appendix B).

\subsection{Student's results in relation to the ECDL standard and the require- ments for passing the exam}

Two additional analyses were done in order to estimate the degree of the students' familiarity with the basic Internet concepts: we tested how many of them would meet the condition for passing the test from the corresponding ECDL base module (M2-Online Essentials) and how many students would fail the exam (answer correctly to less than $51 \%$ of the questions).

The pass mark for the ECDL test is 75\%, which corresponds to 6 correct answers (of 8 questions) in this test. Based on the chart shown in Figure 11, it can be seen that less than $20 \%$ of the students would meet this condition in most of the groups, and in the case of two groups (FAM and HUN) nobody would pass the test. The numbers of students who satisfy this requirement are shown in Table 5 (ECDL row) together with the total number of students in the groups ( $\mathrm{N}$ row). It can be seen from this table that only every fifth first-year student (100 of 522) would meet the ECDL 75\% pass mark, i.e. only around $19 \%$.

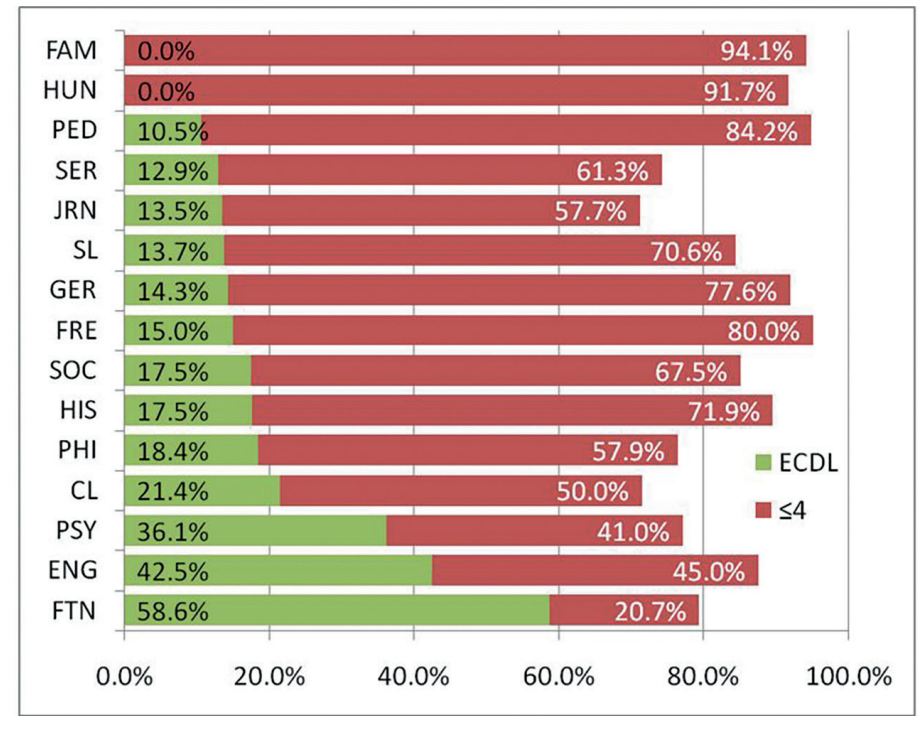

Figure 11. Percentage of students who meet the condition defined by the ECDL standard

(ECDL) and those who answered correctly to less than $51 \%$ of the questions $(\leq 4)$ 
On the other hand, when it comes to the minimum requirements for passing the exam, Figure 11 shows that the percentage of first-year students from the Faculty of Philosophy who would not pass the exam ranged from $45 \%$ in the ENG group (18 out of 40 students) to about $92 \%$ in the HUN group (11 out of 12 students). The numbers of those students for each group are given in the bottom row of Table 5 marked with $\leq 4$. These data show that nearly $65 \%$ (338 out of 522) of the first-year students from the FF who were tested would not pass the exam (according to the faculties' criteria).

\begin{tabular}{|c|c|c|c|c|c|c|c|c|c|c|c|c|c|c|c|c|c|}
\hline & JRN & HIS & PSY & HUN & PHI & soc & CL & SER & GER & ENG & FRE & PED & SL & FTN & FAM & FF & ALL \\
\hline $\mathrm{N}$ & 52 & 57 & 61 & 12 & 38 & 40 & 14 & 31 & 49 & 40 & 20 & 57 & 51 & 29 & 17 & 522 & 568 \\
\hline ECDL & 7 & 10 & 22 & 0 & 7 & 7 & 3 & 4 & 7 & 17 & 3 & 6 & 7 & 17 & 0 & 100 & 117 \\
\hline$\leq 4$ & 30 & 41 & 25 & 11 & 22 & 27 & 7 & 19 & 38 & 18 & 16 & 48 & 36 & 6 & 16 & 338 & 360 \\
\hline
\end{tabular}

Table 5. Total number of students who participated in the research $(\mathrm{N})$, number of students who answered correctly more than 5 questions (ECDL), and number of students who answered correctly fewer than 5 questions $(\leq 4)$

\subsection{Correlations between the questions}

Based on the correlation coefficients between test questions calculated on the whole sample (Table 6), it can be seen that the correlations between questions were very low (compared to the maximum value of 1 ). This indicates that, although the test measured knowledge of the same field, the students answered the questions quite inconsistently - a correct answer to one question does not mean that a student will give correct answers to the other questions as well. The question about the Internet (INET) was the least related to the others, i.e. the correctness of answer to this question was not related to the correctness of the answers to any other question. The question about URL was related only to the question about the domain (ORG) with a correlation of only 0.124 . The highest correlation was between the questions about MAIL and domain (ORG), but this was also quite low (0.230).

For the sample of students from the FF, who made up majority of the sample, the relations between the questions were approximately the same as in the total sample (Table 8 in Appendix B). On the other hand, the relations were somewhat different in the subsamples of FTN and FAM (Tables 9 and 10 in Appendix B), primarily because fewer numbers of correlation coefficients met the significance criterion, which was probably because they were calculated on a smaller sample. For the sample of FTN students, the question about the Internet (INET) showed a significant relation with the questions about IP and HTML. However, for the FAM sample, significant correlations were rare among the questions and they had negative signs (a correct answer to one question is related to a wrong answer for another). Although significant, these relations were possibly a consequence of some random result variations due to the small number of respondents. 


\begin{tabular}{|c|c|c|c|c|c|c|c|c|}
\hline & INET & IP & LAN & URL & ORG & HTML & IE & MAIL \\
\hline INET & & 0.073 & 0.062 & 0.028 & -0.033 & 0.061 & 0.037 & -0.036 \\
\hline IP & & & 0.178 & 0.022 & 0.178 & 0.125 & 0.096 & 0.137 \\
\hline LAN & & & & 0.045 & 0.159 & 0.136 & 0.178 & 0.151 \\
\hline URL & & & & & 0.124 & 0.078 & 0.045 & 0.066 \\
\hline ORG & & & & & & 0.138 & 0.147 & 0.230 \\
\hline HTML & & & & & & & 0.084 & 0.103 \\
\hline IE & & & & & & & & 0.099 \\
\hline MAIL & & & & & & & & \\
\hline
\end{tabular}

Note: Bold values are significant at the level 0.05

Table 6. Correlation coefficients (phi) between individual questions for the total sample of respondents

\section{Discussion}

The research presented in this paper was carried out with the aim of assessing the pre-knowledge of non-ICT first-year students at the Faculty of Philosophy at the University of Novi Sad about the key concepts in computer literacy. Contrary to the general assumption that the knowledge of digital natives is adequate and up to date, the author's experience in teaching suggested that they are generally not sufficiently familiar even with the basic concepts. The freshmen's knowledge was consequently evaluated in the present study relying on a test consisting of 22 close-ended questions with 4 options per question. The questions were selected from the set of questions on which the Computer Literacy exam is based. The student's answers to 8 questions related to the Internet were analysed in this paper.

In accordance with previous research (Heerwegh, De Wit and C. Verhoeven 2016; O'Hanlon 2002; van Deursen and van Diepen 2013) and with the authors' own in-classroom experience, the results confirmed the relatively poor familiarity of first-year students with basic Internet concepts at the beginning of their studies: the percentage of correct and incorrect answers of the FF freshmen was about 50\%. The percentage of correct answers at the level of individual study groups of the FF ranged between $36.5 \%$ (HUN) and $64.4 \%$ (ENG), and the average number of correct answers varied between 2.92 (HUN) and 5.15 (ENG). The highest homogeneity of accurate answers was detected in the case of the question about web browsers (IE), and the lowest one in the case of the question about URL.

Regarding the number of correct answers, when observing all the analysed study groups (including the two control groups FTN and FAM), statistically significant differences were detected between a number of them. These differences may be explained by: 
- The type of study programme - students who enrolled on technical studies (FTN) showed richer knowledge; here, they had probably been more interested in the field of information/computer science before their studies compared to the FF and FAM students.

- For the FF, the ENG and PSY students had the best results, because those are the study groups with the strictest selection on the entrance exam due to the large number of candidates. Apart from high results in the entrance exam, the students in those groups probably had a high grade-average in high school.

The analysis of the freshmen's results in relation to the ECDL standard (the percentage of students who met the condition defined by the ECDL standard was between $0 \%$ (HUN) and $42.5 \%$ (ENG)) and the fulfilment of the requirements for passing the exam (the percentage of students who would fail the exam was between 91.7\% (HUN) and 45\% (ENG)) further highlights the need to improve their computer literacy.

The results of this research suggest that the familiarity of the first-year students at the Faculty of Philosophy at the University of Novi Sad with the basic concepts of Internet is unsystematic, incomplete, and rather uneven. This is probably partly due to the unequal coverage of computer literacy in secondary education and to the differences in students' preferences. These findings emphasise the need for long-term monitoring of the freshmen's pre-knowledge and the necessity for systematising their knowledge at the beginning of their studies through appropriate basic subjects in computer literacy. In order to gain insights into the general state of computer literacy among students, it would be preferable to expand the research to other higher educational institutions, and in addition to assessing their familiarity with the basic concepts, their practical skills should also be tested. 


\section{References}

Deniz, Müjgan Hacıoğlu, and Seda Karakaş Geyik. "An Empirical Research on General Internet Usage Patterns of Undergraduate Students.” Procedia - Social and Behavioral Sciences 195 (July 2015): 895-904.

https://doi.org/10.1016/j.sbspro.2015.06.369

Duţă, Nicoleta, and Oscar Martínez-Rivera. "Between Theory and Practice: The Importance of ICT in Higher Education as a Tool for Collaborative Learning.” Procedia - Social and Behavioral Sciences 180 (May 2015): 1466-73.

https://doi.org/10.1016/j.sbspro.2015.02.294

Heerwegh, Dirk, Kurt De Wit, and Jef C. Verhoeven. "Exploring the Self-Reported ICT Skill Levels of Undergraduate Science Students.” Journal of Information Technology Education: Research 15 (2016): 19-47.

https://doi.org/10.28945/2334

Jokić, Andrea, Dragana Koljenik, Sanjica Tanacković Faletar, and Boris Badurina. "Vještine Informacijske i Informatičke Pismenosti Studenata Informacijskih Znanosti u Osijeku: Pilot-Istraživanje.” Vjesnik Bibliotekara Hrvatske 59, No. 3-4 (2016): 63-92.

https://hrcak.srce.hr/187610

Khairnar, C. M. "Advance Pedagogy: Innovative Methods of Teaching and Learning." International Journal of Information and Education Technology 5, No. 11 (2015): 869-72. https://doi.org/10.7763/IjIET.2015.V5.629

Lekka, Aspa, and Jenny Pange. "What ICT Tools Do Undergraduate Students Use?” In 2015 International Conference on Interactive Mobile Communication Technologies and Learning (IMCL), 386-88. IEEE, 2015.

https://doi.org/10.1109/IMCTL.2015.7359625

Molnár, Gyöngyvér. "The Use of Innovative Tools in Teacher Education: A Case Study.” In Proceedings of the Fifth International Conference on Informatics, Educational Technology and New Media in Education, 44-49. Sombor: Faculty of Education, 2008.

http://www.staff.u-szeged.hu/ gymolnar/sombor 2.pdf

O’Hanlon, Nancy. "Net Knowledge: Performance of New College Students on an Internet Skills Proficiency Test.” The Internet and Higher Education 5, No. 1 (2002): 55-66.

https://doi.org/10.1016/S1096-7516(02)00066-0

Prensky, Marc. “Digital Natives, Digital Immigrants Part 1.” On the Horizon 9, No. 5 (2001a): 1-6. https://doi.org/10.1108/10748120110424816

Prensky, Marc. “Digital Natives, Digital Immigrants Part 2: Do They Really Think Differently?” On the Horizon 9, No. 6 (2001b): 1-6 https://doi.org/10.1108/10748120110424843

Serdyukov, Peter. “Innovation in Education: What Works, What Doesn't, and What to Do about It?” Journal of Research in Innovative Teaching \& Learning 10, No. 1 (2017): 4-33. https://doi.org/10.1108/JRIT-10-2016-0007

Slechtova, Pavla. "Attitudes of Undergraduate Students to the Use of ICT in Education." Procedia - Social and Behavioral Sciences 171 (January, 2015): 1128-34.

https://doi.org/10.1016/j.sbspro.2015.01.218

Valić Nedeljković, Dubravka. “Deca, Mediji i „trošenje Budžeta“ Slobodnog Vremena.” Medijski Dijalozi IV, No. 10 (2011): 339-54. 
Valić Nedeljković, Dubravka. “Deca i Zadovoljavanje Informativnih Potreba.” Medijski Dijalozi V, No. 11 (2012): 325-36.

Valić Nedeljković, Dubravka, Karlo Bala, and Zoltan Geler. "Deca u Virtuelnom Svetu Kompjuterskih Igara.” In Digitalne Medijske Tehnologije i Društveno-Obrazovne Promene, Knjiga 3, edited by Dubravka Valić Nedeljković, and Dejan Pralica, 241-54. Novi Sad: Filozofski fakultet, 2013.

Van Deursen, A.J.A.M., and S. van Diepen. "Information and Strategic Internet Skills of Secondary Students: A Performance Test.” Computers \& Education 63 (April 2013): 218-26. https://doi.org/10.1016/j.compedu.2012.12.007 


\section{Appendix A. Test questions}

1. The Internet is

1. World Wide Web

2. global computer network

3. social network

4. type of server

3. LAN is a computer network which

1. covers a wider area (e.g. town, region)

2. covers a relatively small area (e.g. office, classroom, building)

3. connects distant servers

4. is a global computer network

5. Choose the top-level domain which is used for websites of non-profit organisations

1. COM

2. NET

3. ORG

4. XLSX

7. Choose the program which is a Web browser

1. Google Mail

2. Windows Explorer

3. Internet Explorer

4. Microsoft FrontPage
2. IP stands for

1. Internet Store (Ser. Internet Prodavnica)

2. Internet Provider

3. Internet Protocol

4. Internet Presentation

4. URL is

1. transfer protocol for web pages

2. numerical label that uniquely identifies every computer in a network

3. domain name

4. unique address of Internet resources

6. The language used to make Web pages is called

1. World Wide Web

2. HyperText Markup Language

3. HTTP

4. Hypertext

8. Choose the program used for sending and receiving electronic mail

1. Thunderbird

2. Chrome

3. Google Talk

4. Twitter 


\section{Appendix B. The results of additional statistical tests}

\begin{tabular}{|c|c|c|c|c|c|c|c|c|c|c|c|c|c|c|c|}
\hline & FTN & ENG & PSY & CL & FRE & PHI & JRN & SER & soc & GER & SL & HIS & FAM & PED & HUN \\
\hline FTN & & & & & & 0.175 & 0.091 & 0.054 & 0.009 & 0.001 & 0.001 & 0.000 & 0.001 & 0.000 & 0.001 \\
\hline ENG & & & & & & & & & 0.413 & 0.052 & 0.087 & 0.009 & 0.021 & 0.000 & 0.026 \\
\hline PSY & & & & & & & & & 0.987 & 0.115 & 0.197 & 0.017 & 0.050 & 0.000 & 0.058 \\
\hline CL & & & & & & & & & & & & & & 0.543 & \\
\hline FRE & & & & & & & & & & & & & & 0.367 & \\
\hline PHI & & & & & & & & & & & & & & 0.261 & \\
\hline JRN & & & & & & & & & & & & & & 0.094 & \\
\hline SER & & & & & & & & & & & & & & & \\
\hline SOC & & & & & & & & & & & & & & & \\
\hline GER & & & & & & & & & & & & & & & \\
\hline SL & & & & & & & & & & & & & & & \\
\hline HIS & & & & & & & & & & & & & & & \\
\hline FAM & & & & & & & & & & & & & & & \\
\hline PED & & & & & & & & & & & & & & & \\
\hline HUN & & & & & & & & & & & & & & & \\
\hline
\end{tabular}

Note: Only p values lower than 1 are shown, bold values are significant at level 0.05

Table 7. Significance of z-tests of contrasting between mean ranges of results of the analysed study programmes expressed in $\mathrm{p}$ levels

\begin{tabular}{|c|c|c|c|c|c|c|c|c|}
\hline & INET & IP & LAN & URL & ORG & HTML & IE & MAIL \\
\hline INET & & 0.035 & 0.032 & 0.032 & -0.036 & 0.020 & 0.051 & -0.052 \\
\hline IP & & & 0.160 & 0.035 & 0.162 & 0.082 & 0.095 & 0.129 \\
\hline LAN & & & & 0.054 & 0.163 & 0.119 & 0.184 & 0.146 \\
\hline URL & & & & & 0.113 & 0.081 & 0.033 & 0.063 \\
\hline ORG & & & & & & 0.142 & 0.140 & 0.226 \\
\hline HTML & & & & & & & 0.088 & 0.101 \\
\hline IE & & & & & & & & 0.112 \\
\hline MAIL & & & & & & & & \\
\hline
\end{tabular}

Note: Bold values are significant at level 0.05

Table 8. Correlation coefficients (phi) between individual questions on the sample of students from FF 


\begin{tabular}{|c|c|c|c|c|c|c|c|c|}
\hline & INET & IP & LAN & URL & ORG & HTML & IE & MAII \\
\hline INET & & 0.508 & 0.289 & -0.012 & 0.053 & 0.478 & -0.097 & 0.118 \\
\hline IP & & & 0.475 & -0.070 & 0.302 & 0.555 & -0.107 & 0.346 \\
\hline LAN & & & & -0.290 & 0.243 & 0.099 & -0.076 & 0.159 \\
\hline URL & & & & & 0.367 & -0.119 & 0.260 & 0.070 \\
\hline ORG & & & & & & 0.010 & 0.210 & 0.255 \\
\hline HTML & & & & & & & -0.148 & -0.010 \\
\hline IE & & & & & & & & -0.210 \\
\hline MAIL & & & & & & & & \\
\hline
\end{tabular}

Note: Bold values are significant at level 0.05

Table 9. Correlation coefficients (phi) between individual questions on the sample of FTN students

\begin{tabular}{|c|c|c|c|c|c|c|c|c|}
\hline & INET & IP & LAN & URL & ORG & HTML & IE & MAIL \\
\hline INET & & 0.033 & 0.310 & -0.181 & -0.436 & 0.182 & -0.491 & 0.033 \\
\hline IP & & & -0.381 & -0.523 & 0.107 & -0.257 & -0.107 & -0.308 \\
\hline LAN & & & & 0.070 & -0.240 & 0.074 & -0.074 & 0.182 \\
\hline URL & & & & & -0.127 & 0.182 & 0.127 & 0.033 \\
\hline ORG & & & & & & -0.214 & 0.214 & 0.107 \\
\hline HTML & & & & & & & -0.190 & 0.107 \\
\hline IE & & & & & & & & -0.107 \\
\hline MAIL & & & & & & & & \\
\hline
\end{tabular}

Note: Bold values are significant at level 0.05

Table 10. Correlation coefficients (phi) between individual questions on the sample of FAM students 